\title{
Rapid Sequencing of Multiple RNA Viruses in Their Native Form
}

Thidathip Wongsurawat ${ }^{1 \dagger}$, Piroon Jenjaroenpun ${ }^{1 \dagger}$, Mariah K. Taylor ${ }^{2}$, Jasper Lee ${ }^{2}$, Aline Lavado Tolardo ${ }^{3}$, Jyothi Parvathareddy4, Sangam Kandel'1,5, Taylor D. Wadley', Bualan Kaewnapan ${ }^{6}$, Niracha Athipanyasilp ${ }^{6}$, Andrew Skidmore ${ }^{7}$, Donghoon Chung ${ }^{7}$, Chutikarn Chaimayo ${ }^{6}$, Michael Whitt ${ }^{2}$, Wannee Kantakamalakul', Ruengpung Sutthent ${ }^{6}$, Navin Horthongkham ${ }^{6}$, David W. Ussery ${ }^{1,8}$, Colleen B. Jonsson ${ }^{2}$ and Intawat Nookaew ${ }^{1,8 *}$

\section{OPEN ACCESS}

Edited by:

Sead Sabanadzovic,

Mississippi State University,

United States

Reviewed by:

Timokratis Karamitros, University of Oxford, United Kingdom

Pasquale Saldarelli,

Institute for Sustainable Plant

Protection (IPSP-CNR), Italy

*Correspondence:

Intawat Nookaew

inookaew@uams.edu

${ }^{\dagger}$ These authors have contributed equally to this work

Specialty section: This article was submitted to Virology,

a section of the journa

Frontiers in Microbiology

Received: 18 November 2018 Accepted: 31 January 2019

Published: 25 February 2019

Citation:

Wongsurawat $T$, Jenjaroenpun $P$, Taylor MK, Lee J, Tolardo AL,

Parvathareddy J, Kandel S,

Wadley TD, Kaewnapan B,

Athipanyasilp N, Skidmore A

Chung D, Chaimayo C, Whitt $M$, Kantakamalakul W, Sutthent $R$,

Horthongkham N, Ussery DW, Jonsson CB and Nookaew I (2019) Rapid Sequencing of Multiple RNA

Viruses in Their Native Form.

Front. Microbiol. 10:260.

doi: 10.3389/fmicb.2019.00260
${ }^{1}$ Department of Biomedical Informatics, College of Medicine, University of Arkansas for Medical Sciences, Little Rock, AR United States, ${ }^{2}$ Department of Microbiology, Immunology and Biochemistry, The University of Tennessee Health Science Center, Memphis, TN, United States, ${ }^{3}$ Virology Research Center, Ribeirão Preto Medical School, University of São Paulo, Ribeirão Preto, Brazil, ${ }^{4}$ Regional Biocontainment Laboratory, University of Tennessee Health Science Center, Memphis, TN, United States, ${ }^{5}$ Department of Bioinformatics, University of Arkansas at Little Rock, Little Rock, AR, United States, ${ }^{6}$ Department of Microbiology, Faculty of Medicine Siriraj Hospital, Mahidol University, Bangkok, Thailand, ${ }^{7}$ Department of Microbiology and Immunology, University of Louisville, Louisville, KY, United States, ${ }^{8}$ Department of Physiology and Biophysics, College of Medicine, University of Arkansas for Medical Sciences, Little Rock, AR, United States

Long-read nanopore sequencing by a MinION device offers the unique possibility to directly sequence native RNA. We combined an enzymatic poly-A tailing reaction with the native RNA sequencing to (i) sequence complex population of singlestranded (ss)RNA viruses in parallel, (ii) detect genome, subgenomic mRNA/mRNA simultaneously, (iii) detect a complex transcriptomic architecture without the need for assembly, (iv) enable real-time detection. Using this protocol, positive-ssRNA, negativessRNA, with/without a poly(A)-tail, segmented/non-segmented genomes were mixed and sequenced in parallel. Mapping of the generated sequences on the reference genomes showed $100 \%$ length recovery with up to $97 \%$ identity. This work provides a proof of principle and the validity of this strategy, opening up a wide range of applications to study RNA viruses.

Keywords: native RNA, genome, subgenomic mRNA, single-stranded RNA, virus, nanopore sequencing, rapid detection, MinION

\section{INTRODUCTION}

Infectious disease epidemics are primarily driven by RNA viruses (Woolhouse et al., 2016; Carrasco-Hernandez et al., 2017) and hence are likely agent of future pandemics (CarrascoHernandez et al., 2017). Due to the lack of proofreading in RNA polymerases, RNA viruses are error-prone during genome replication, providing a platform for rapid evolution in new environments and hosts. Currently, genome sequences of many viruses are available and served as a powerful resource for molecular surveillance, pathogen characterization, diagnosis, and antiviral drug discovery (DeFilippis et al., 2003). Still, outbreaks of viral diseases are a neverending challenge. Short-read sequencing methods to recover RNA viral genomes relies on reverse transcription (RT) of RNA to cDNA which requires primer optimization and amplification. These steps introduce many biases, artifacts and make rapid diagnosis difficult (Marston et al., 2013). Alternatively, real-time sequencing using a pocket-sized MinION device Oxford Nanopore Technologies (ONT) skips these steps and makes rapid detection and characterization of emerging 
pathogens possible (Hoenen et al., 2016; Kilianski et al., 2016; Faria et al., 2017). A shorter procedure on the MinION by elimination of cDNA synthesis step can be accomplished through direct RNA sequencing (dRNA-seq), designed to directly ligate and sequence only polyadenylated RNA, providing strand-specific information and base modifications of RNA (Garalde et al., 2018; Jenjaroenpun et al., 2018). Independent from the poly(A) enrichment, a sequence-specific $3^{\prime}$-adapter for targeting non-polyadenylated RNA can be designed and has been successfully demonstrated (Garalde et al., 2018) and influenza A virus (Keller et al., 2018). Moreover, target sequence capture/hybridization techniques could be applied before sequencing library preparation to enrich viral genetic materials (Dapprich et al., 2016; Eckert et al., 2016; Karamitros and Magiorkinis, 2018). These methods can reduce background noise from host RNAs. However, designing sequence-specific 3 -adaptor or probing sequence needs a prior knowledge of a nucleotide of the target as prerequisite. Therefore, novel RNA viruses, or coinfections might not be discovered by this approach.

We established a rapid procedure and evaluated the feasibility of MinION for complete sequencing of multiple, single-stranded RNA (ssRNA) viruses in a single reaction to obtain complete genomes. Our approach combines (1) poly(A)-tailing using simple enzymatic reaction, (2) native RNA sequencing by MinION, and (3) real-time analysis aiming to simultaneously detect and characterize multiple RNA viruses. A mix of negative( -$)$ and positive(+) ssRNA viruses, including Mayaro virus (MAYV), Venezuelan equine encephalitis virus (VEEV), Chikungunya virus (CHIKV), Zika virus (ZIKV), vesicular stomatitis Indiana virus (VSV), and Oropouche virus (OROV) were used in this demonstration.

\section{RESULTS}

To establish an optimal procedure for real-time native RNA sequencing, we performed three experiments. 1) Sequencing two polyadenylated RNA viruses without RT, suggested by the original protocol, 2) Adding poly(A)-tail to non-polyadenylated RNA phage and ZIKVs using E. coli poly(A) polymerase (EPAP), followed by sequencing, and 3) Applying ribosomal RNA (rRNA) depletion step to host-rRNA contaminated samples, followed by poly(A)-tailing and sequencing. Then, we applied the optimized protocol on a mix of all RNA viruses.

In the first scenario, naturally polyadenylated $(+)$ ssRNA alphaviruses, MAYV and VEEV, genome size approximately $11.5 \mathrm{~kb}$, were used. Extracted RNA (see section "Materials and Methods") of MAYV and VEEV were pooled at approximately the same concentration $(\sim 350 \mathrm{ng})$ and used for library preparation of the dRNA-seq protocol, following the manufacturer's recommendations, except the RT step was skipped (see section "Materials and Methods"). The prepared library then was loaded into ONT flow cell and sequenced. The sequenced reads were mapped on the genomes and analyzed (Table 1), resulting in full genome coverage of the both viruses with 716X and 8184X, for MAYV and VEEV, respectively. We obtained $97 \%$ sequence identity by consensus polishing. A high viral/host ratio of 21 was observed because the most abundant RNA species in host, rRNAs, were not captured. By this scenario, multiple viral whole genome sequencing of polyadenylated viruses without RT is possible.

The second scenario sought to evaluate the feasibility of using the poly(A)-tailing reaction coupled with dRNA-seq, $(+)$ ssRNA. Bacteriophage MS2 was used first. Five hundred nanograms of $3.57 \mathrm{~kb}$ RNA was treated with EPAP using an optimized poly(A)tailing reaction (see section "Materials and Methods"). Poly(A)tailed RNA was then used as input for library preparation and sequencing in the same way as described above, resulting in a complete read of the bacteriophage genome (Table 1). We then applied the procedure on ZIKV RNA, non-polyadenylated $(+)$ ssRNA with $\sim 11 \mathrm{~kb}$ length, and obtained long-read. In both scenarios, we observed reduced coverage at the $5^{\prime}$ and a heavy coverage bias towards the $3^{\prime}$, however, we found reads that almost spanned the entire genome (Figure 1B). By poly(A)-tailing reaction, the majority of RNA in ZIKV sample was host RNA that could be captured after EPAP treatment resulted very low virus/host ratio of 0.0014 . By this scenario, we obtained a rapid procedure to universally detect ssRNA viruses in real-time. The workflow of poly(A)-tailing plus dRNA-seq was accomplished and summarized in Figure 1A. The host RNA contamination problem was taken into account in the next scenario.

In the third scenario, we introduced host rRNA depletion step using a rRNA removal kit, to increase the amount of viral RNA reads. The rRNA depletion was performed before poly(A)-tailing and dRNA-seq. The ZIKV sample was used to evaluate changes in ratio of viral/host RNA. We found that introducing the depletion step resulted in a 160-fold increase in proportion of viral RNA reads/ host reads compared to the nondepleted sequencing. However, we observed high proportion of low-quality sequences compared to non-depleted scenario. We further tested the impact on rRNA depletion step on artificial mixing of $2 \%$ of bacteriophage MS2 RNA and 98\% of total RNA extracted from mouse cells. The rRNA depletion kit was used for the mixture as described above. Again, the proportion of low quality reads was increased as seen in rRNA-depleted ZIKV sample (Table 1). This problem may be associated with the remained compound from the rRNA depletion process that interfere the dRNA-seq chemistry. Therefore, the depletion step need to be further optimized, we decided not to use the depletion step in our protocol, as it may add complexity and increase the low-quality sequences.

Finally, we applied the established procedure to sequence a pool of six RNA viruses including MAYV, VEEV, CHIKV, ZIKV, VSV, and OROV, which were each purified from supernatant of varying cell lines and RNA was extracted from purified virions (see section "Materials and Methods"). This mixed RNA viruses has diversified genomic property i.e., (+)ssRNA, (-)ssRNA, lack/presence of poly(A)-tail, segmented/non-segmented genomes (Table 2). The RNA input from each sample varied from 16 to $66 \mathrm{ng}$ (215 ng in total) (Table 2). Sequencing on MinION was performed for $\sim 8 \mathrm{~h}$ in total with real-time base calling, enabling data analysis in real-time (see section "Materials and Methods"). The sequences were mapped to the viral genomes and host genomes (Table 2). Remarkably, within the first two 
minutes, the first reads of all six viruses were translocated through nanopore (Figure 1C). In only two hours, complete or near complete genomes of all the six viruses were acquired (Figure 1D). In total 85,711 reads were generated, 5,000 (6\%) were mapped on viruses, 62,060 (72\%) were mapped on host genomes, and 18,651 (22\%) were unclassified (low quality). Mapping of the 5,000 reads on the six genomes showed $100 \%$ recovery of the MAYV, VEEV, CHIKV, VSV, and OROV at 48X $659 \mathrm{X}$ coverage and showed $99 \%$ recovery of the ZIKV, the least abundance in the mix, at 10X coverage (Table 2). We obtained several long reads $(>10 \mathrm{~kb}$ ) that covered $>95 \%$ of MAYV, VEEV, and VSV genomes while the longest reads mapped to CHIKV and ZIKV were 84 and $68 \%$ of genome length, respectively. Unlike the other viruses, OROV has a tripartite genome of small $(\mathrm{S})$, medium $(\mathrm{M})$, and large (L) segments. We found the longest read covered $99 \%$ of $S(0.96 \mathrm{~kb}), 98 \%$ of $\mathrm{M}$ and $\mathrm{L}(4.39 \mathrm{~kb}$ and $6.85 \mathrm{~kb})$. Notably, although high error rates of individual native RNA sequence were observed ( $\sim 82 \%$ read identity), $93-97 \%$ identity can be achieved by consensus polishing.

We inspected the mapped reads based on strand-orientation and found feasibility of simultaneous detection of viral genome and its transcripts (Figure 2). We observed the two spikes of read coverage depth around the gap of two subgenomic mRNA of VEEV on the coverage plot (Figure 2A) clearly indicating that the two subgenomic mRNAs were independently transcribed and the reads that spanned over the gap belonged to the genomic material (see section "Materials and Methods"). This result is based on sequencing RNA directly without primer bias, RT and amplification. However, observation of two subgenomic mRNA in VEEV need further deep investigation. Conversely, (-)ssRNA virus needs to transcribe mRNA in the opposite direction of the genome. We found mapped reads derived from the mRNA and genome of OROV nicely aligned in opposite direction (Figure 2B).

\section{DISCUSSION}

This versatile protocol can contribute viral RNA research by (i) retaining the strand direction; this facilitates simultaneously detection of genome, subgenomic mRNA/mRNA, recombinant, complementary RNA intermediates (ii) increasing the accuracy of determining transcriptomic architecture and recombination with no need for assembly (iii) providing a fast and easy method to perform direct sequencing of any RNA virus (iv) directly sequencing of novel RNA viruses with no specific primer required, including novel ones and (v) saving on the budget (see Table 3). In addition, the native RNA signal can be used for further detection of RNA base modifications (RNA epigenetics) that are known to control viral replication during host infection (Lichinchi et al., 2016). The RNA base modifications, occurred on native RNA sequence, alter the ionic current signal recorded

TABLE 1 | Three optimization scenario.

\begin{tabular}{|c|c|c|c|c|c|c|c|c|c|c|c|}
\hline Scenario & Virus & $\begin{array}{l}\text { Genome } \\
\text { size (kb) }\end{array}$ & $\begin{array}{c}\text { RNA input } \\
\text { (ng in } \\
\text { total) }\end{array}$ & Coverage & $\begin{array}{c}\text { Mean } \\
\text { coverage }\end{array}$ & $\begin{array}{c}\text { Longest } \\
\text { mapped } \\
\text { length (kb) }\end{array}$ & $\begin{array}{l}\text { Mapped } \\
\text { virus (\%) }\end{array}$ & $\begin{array}{l}\text { Mapped } \\
\text { host, \% } \\
\text { (host } \\
\text { rRNA) }\end{array}$ & $\begin{array}{c}\text { Low } \\
\text { quality } \\
\text { sequence } \\
(\%)\end{array}$ & $\begin{array}{l}\text { virus/host } \\
\text { (fold) }\end{array}$ & $\begin{array}{l}\text { Total } \\
\text { number of } \\
\text { reads }\end{array}$ \\
\hline \multirow[t]{2}{*}{$\begin{array}{l}\text { Multiple viral } \\
\text { detection }\end{array}$} & MAYVa & 11.14 & 350 & $100 \%$ & $716 x$ & 11.13 & 6.35 & $4.13(0.41)$ & 8.45 & 21.1740 & 92688 \\
\hline & VEEVa & 11.45 & 350 & $100 \%$ & $8184 \times$ & 11.38 & 81.07 & & & & \\
\hline \multirow[t]{2}{*}{$\begin{array}{l}\text { An enzymatic } \\
\text { reaction for } \\
\text { non-poly(A)-tail } \\
\text { viral RNA }\end{array}$} & phage MS2 & 3.57 & 500 & $100 \%$ & $2887 \times$ & 3.54 & 71.58 & $0(0)$ & 28.42 & NA & 9553 \\
\hline & ZIKV & 10.79 & 54 & $100 \%$ & $4 \times$ & 10.32 & 0.11 & $\begin{array}{c}81.92 \\
(71.32)\end{array}$ & 17.97 & 0.0014 & 16793 \\
\hline \multirow[t]{2}{*}{$\begin{array}{l}\text { Host rRNA } \\
\text { depletion and } \\
\text { enzymatic } \\
\text { reaction for } \\
\text { non-poly(A)-tail } \\
\text { viral RNA }\end{array}$} & ZIKV & 10.79 & 48 & $100 \%$ & $12 \times$ & 10.53 & 3.98 & $\begin{array}{l}17.59 \\
(3.32)\end{array}$ & 78.43 & 0.2264 & 904 \\
\hline & $\begin{array}{l}2 \% \text { phage } \\
\text { MS2 + } 98 \% \\
\text { mouse total } \\
\text { RNA }\end{array}$ & 3.57 & $\begin{array}{c}60 \text { (phage), } \\
2940 \\
\text { (mouse) }\end{array}$ & $100 \%$ & $426 x$ & 3.47 & 3.78 & $\begin{array}{l}34.93 \\
(0.35)\end{array}$ & 61.29 & 0.1083 & 32984 \\
\hline
\end{tabular}

MAY, Mayaro virus; VEEV, Venezuelan equine encephalitis virus; ZIKV, Zika virus. ${ }^{a}$ Presence of polyadenylated genome. 
(1) Poly(A)-tailing reaction

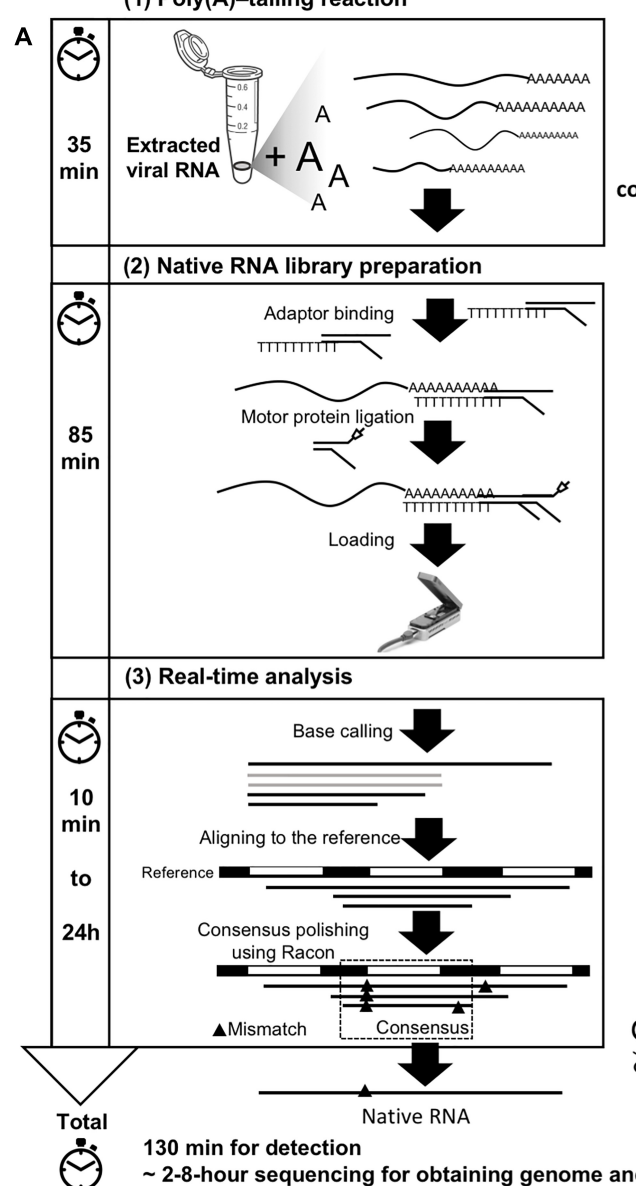

B
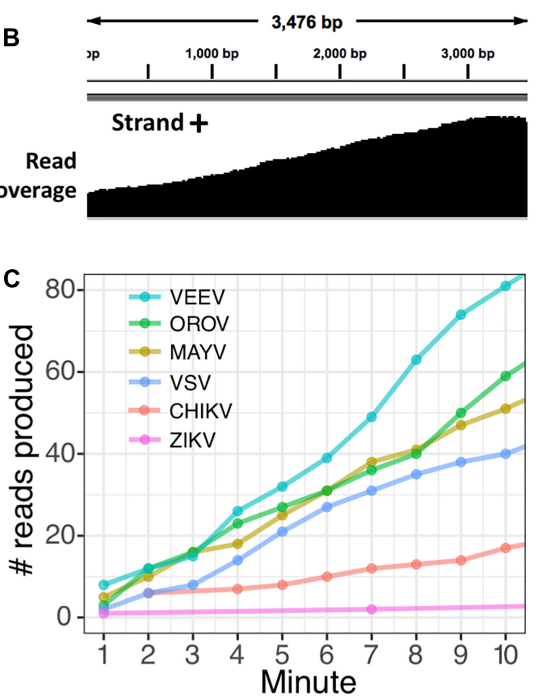

D

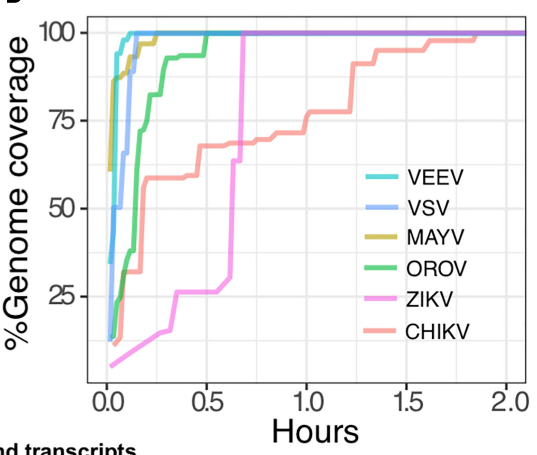

FIGURE 1 | Poly(A)-tailing native RNA-seq protocol. (A) The protocol contains three main steps 1) Poly(A)- tailing reaction using E. coli poly(A) polymerase (EPAP) 2) native RNA-seq by nanopore MinION and 3) real-time analysis to retrieve native RNA sequence. (B) An example of sequencing result (reads) that mapped to the bacteriophage MS2 genome. (C) For detection purpose, this protocol can provide mapped RNA sequences within the first two minutes after sequencing. (D) To obtain whole genome and mRNAs, 2-8 h sequencing could provide more complete information.

TABLE 2 | List of virus used in this study and sequencing result.

\begin{tabular}{|c|c|c|c|c|c|c|c|c|c|c|}
\hline Virus* & $\begin{array}{l}(+) /(-) \\
\text { ssRNA }\end{array}$ & $\begin{array}{l}\text { Genome } \\
\text { size (Kb) }\end{array}$ & $\begin{array}{l}\text { RNA Input } \\
\text { (ng) }\end{array}$ & $\begin{array}{c}\text { Coverage, } \\
\%\end{array}$ & $\begin{array}{c}\text { Mean } \\
\text { coverage }\end{array}$ & $\begin{array}{c}\text { Longest } \\
\text { mapped } \\
\text { length (kb) }\end{array}$ & $\begin{array}{l}\text { Mapped } \\
\text { virus (\%) }\end{array}$ & $\begin{array}{c}\text { Mapped } \\
\text { host (\%) } \\
\text { (host } \\
\text { rRNA) }\end{array}$ & $\begin{array}{c}\text { Low } \\
\text { quality } \\
\text { sequence } \\
(\%)\end{array}$ & $\begin{array}{c}\text { Total } \\
\text { number of } \\
\text { reads }\end{array}$ \\
\hline
\end{tabular}

\section{Poly(A)-tail genome}

MAYV

VEEV

(+) $\quad 11.14$

CHIKV

(+) $\quad 11.45$

$\begin{array}{ll}32 & 100 \\ 35 & 100 \\ 48 & 100\end{array}$

$57 x$
$218 x$
$49 x$

11.11
11.05
10.09

(+)

12.04

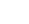

(100

ZIKV

VSV

OROV**

$(+)$

(-)

(-)
10.78

11.16

18

16

$0.96(\mathrm{~S})$

$4.39(\mathrm{M})$

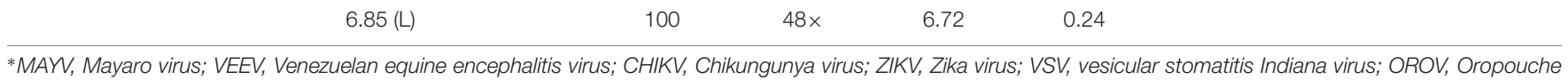

100
100
100
100
100

$10 \times$

7.29

72.40

(32.92)

21.76

85711 virus. **Segmented genome: S, small segment; $M$, medium segment; L, large segment. 


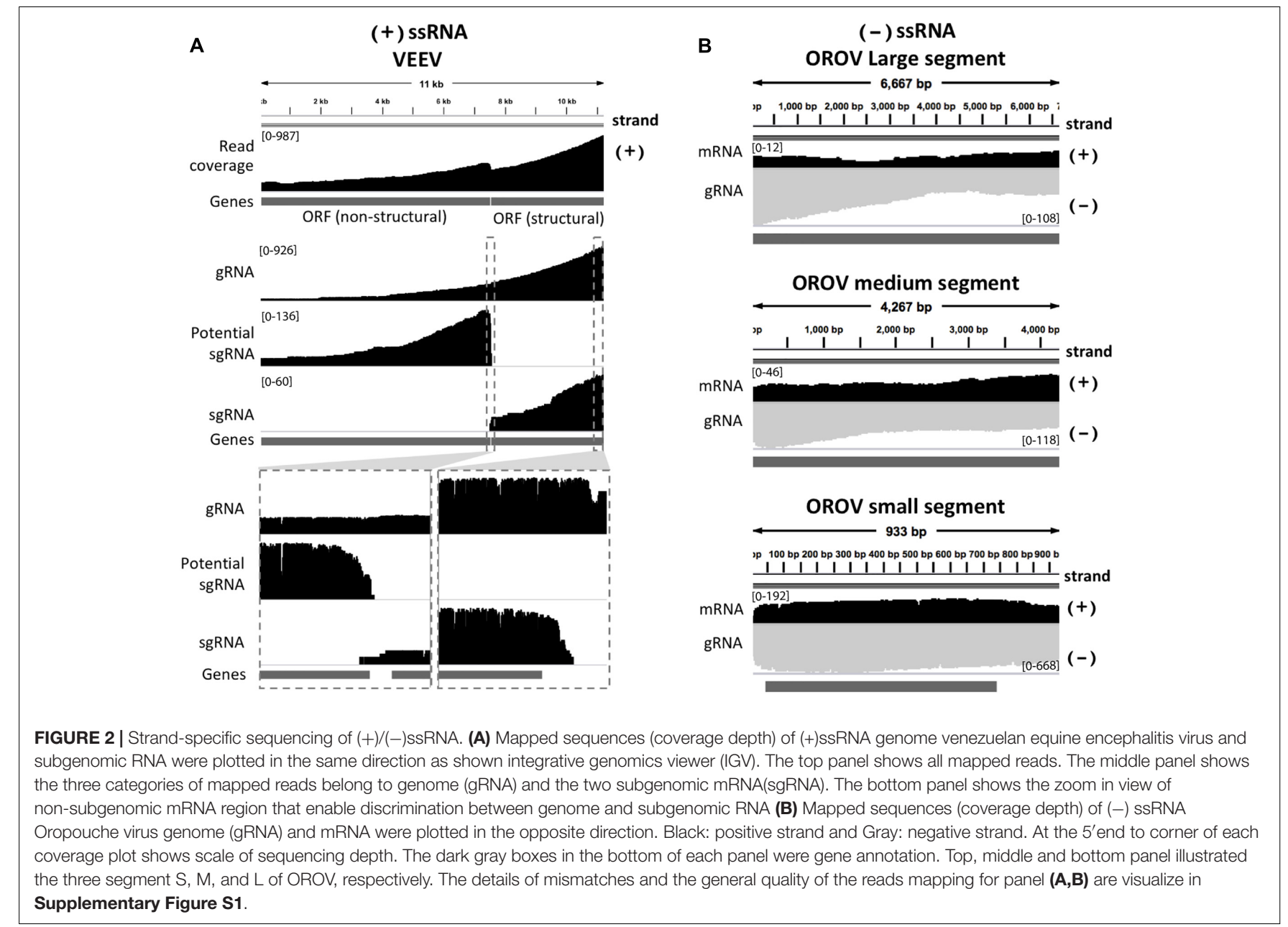

TABLE 3 | Advantages and limitations of this protocol.

\begin{tabular}{|c|c|}
\hline This protocol enables to: & Advantages \\
\hline $\begin{array}{l}\text { (i) detect complex population of RNA viruses simultaneously } \\
\text { whether }(+) \text { ssRNA, (-)ssRNA, lack/presence of poly(A)-tail, } \\
\text { segmented/non-segmented genomes }\end{array}$ & $\begin{array}{l}\text { No specific primer design Co-infection/co-circulation } \\
\text { detection Save on the budget }\end{array}$ \\
\hline $\begin{array}{l}\text { (ii) full-length genomes, subgenomic(sg) mRNAs and } \\
\text { mRNAs }\end{array}$ & $\begin{array}{l}\text { Virus identification Map transcript architecture and } \\
\text { recombination without the need for assembly }\end{array}$ \\
\hline (iii) fast and easy & $\begin{array}{l}\text { Eliminate primer design, reverse transcription, and PCR } \\
\text { bias enabling real-time detection within } 3 \mathrm{~h}\end{array}$ \\
\hline (iv) strand-specific RNA-seq & $\begin{array}{l}\text { Retain the information pertaining to strand origin; facilitate } \\
\text { observation of genome/ transcript/ complementary RNA } \\
\text { strand intermediate. }\end{array}$ \\
\hline (v) sequence native RNA* & Provide signal of RNA base modifications \\
\hline
\end{tabular}

\section{Limitations/ difficulties}

Require high viral load from cultivation Poly(A)-tail reaction is not only specific to viral RNA; host RNA can be a target Because of high error rate, strain level detection is still limited Fully intact RNA and gentle handling are required A loop structure forms at the $3^{\prime}$ terminus might create difficulty for the enzymatic reaction

Only some known modifications are found Deep sequencing is required for detection of RNA modifications and structures (Wongsurawat et al., 2018)

${ }^{*}$ Possible for study RNA epigenetics in the future.

during dRNA-seq (Garalde et al., 2018), leading to the errors on the sequence that is not stochastic (Wongsurawat et al., 2018). By comparison the error of native RNA sequence with its complementary DNA (cDNA) sequence, which contains no error from base modification after RT, multiple RNA modification types can be captured (Wongsurawat et al., 2018). The ability to generate multiple levels of genetic information at high speed has potential to increase our basic understanding of the known 
and undiscovered viruses at a rapid rate. Although promising, however, this protocol carries a number of limitations. First, this protocol requires high amounts of virus. The dRNA-seq detection limit in respect of viral copy number need to be further investigated for clinical application. Second, the poly(A)tail reaction is not only specific to viral RNA; host RNA can also be a target. Competition between host RNAs and viral genome requires a better strategy for host RNAs depletion. Third, although we could identify virus at species-level from error-prone long reads, deep sequencing is required for accurate strainlevel identification. However, improvement of nanopore read accuracy is subject to extensive research, and it is likely that much better accuracy will be available in the future, as well as better depth of viral reads, leading to strain-level identification. In conclusion, our simple protocol could provide rapid viral RNA genome/subgenome sequencing and characterization that can be therefore a versatile tool to study biology of RNA viruses.

\section{MATERIALS AND METHODS}

\section{Viral Cultivation and RNA Extraction Mayaro Virus (MAYV), Oropouche Virus (OROV), and Venezuelan Equine Encephalitis Virus (VEEV)}

Mayaro virus (strain BeAr505411, Genbank No: KP842818.1), OROV (strain BeAn 19991, Genbank No: S: KP052852.1; Segment M: KP052851.1; Segment L: KP052850.1) and VEEV (strain TC-83, Genbank no: L01443.1) were cultivated on Vero cells in MEM with 5\%FBS and 1\%PenStrep. The infection was made on $80 \%$ confluent cells with an MOI of 1 for MAYO and 0.5 for OROV. Both viruses were incubated with cells for 3 days. The viruses were then aliquoted and centrifuged according to protocol. The TRIzol LS extraction (Invitrogen) was carried out following the manufacturer's instruction for RNA extraction with the exception of adding glycogen after separating the aqueous phase from the organic layer and interphase. Purified RNA was resuspended in $50 \mu \mathrm{l}$ RNase-free water and stored at $-70^{\circ} \mathrm{C}$.

\section{Chikungunya Virus (CHIKV)}

CHIKV (strain 181/25 was obtained from BEI resources, Genbank no: EF452493.1) was propagated in Vero 76 cells (ATCC CRL-1587) grown in MEM-E with 10\% FBS. After CPE was monitored, the cell supernatant was harvested and clarified with a low-speed centrifugation at $4,000 \times g$ for $20 \mathrm{~min}$. The cell supernatant was stored at $-80^{\circ} \mathrm{C}$ until use and viral RNA was isolated from the supernatants. RNA was isolated using RNAzol RT (MRC) with the Direct-zol RNA miniprep kit (Zymo Research) according to manufacturer's instructions, with one additional wash step.

\section{Vesicular Stomatitis Indiana Virus (VSV)}

BHK-21 cells were infected at a multiplicity of 0.1 and the culture medium was collected after $22 \mathrm{~h}$. The culture media were first clarified by centrifuging at $8,000 \times g$ for $10 \mathrm{~min}$ then the supernatants were layered on top of a $20 \%$ sucrose cushion and pelleted at $100,000 \times g$ for $1 \mathrm{~h}$. The virus pellet was resuspended in Tris-saline, $\mathrm{pH} 7.4$ and stored at $-80^{\circ} \mathrm{C}$. The viral pellet was subjected to a phenol-chloroform RNA extraction from TRIzol LS (Invitrogen) following manufacturer's protocol with the exception of adding glycogen after separating the aqueous phase from the organic layer and interphase. Purified RNA was resuspended in $20 \mu \mathrm{l}$ RNase-free water and stored at $-70^{\circ} \mathrm{C}$.

\section{Zika Virus (ZIKV)}

Two strains of ZIKV were used for this study.

(1) Genomic RNA of ZIKV (strain MR 766, GenBank: AY632535.2) used for method optimization was purchased from ATCC. ${ }^{1}$

(2) ZIKV (strain PRVABC59 provided by Dr. Barbara Johnson at CDC, GenBank: KU501215) used in pooled viruses sequencing was propagated in Vero 76 cells grown in MEME (GIBCO Laboratories) with $10 \%$ fetal bovine serum. After CPE was monitored, the cell supernatant was harvested and clarified with a low-speed centrifugation at $4,000 \times g$ for $20 \mathrm{~min}$. The cell supernatant was stored at $-80^{\circ} \mathrm{C}$ until use and viral RNA was isolated from the supernatants. RNA was isolated using RNAzol RT (MRC) with the Direct-zol RNA miniprep kit (Zymo Research) according to manufacturer's instructions, with one additional wash step.

\section{Bacteriophage MS2 RNA}

RNA from bacteriophage MS2 (Sigma) is composed of 3569 nucleotides (Genbank no: NC_001417.2). MS2 RNA is free of host nucleic acids and proteins. ${ }^{2}$

\section{Mouse RNA}

RNA isolation was performed from a mouse cell line with the Direct-Zol RNA miniprep Kit (Zymo Research) as per manufacturer's instructions. Total RNA was eluted in $50 \mu \mathrm{l}$ nuclease free water and stored at $-80^{\circ} \mathrm{C}$.

\section{Ribosomal RNA (rRNA) Depletion}

Host cytoplasmic and mitochondrial rRNA was depleted from the samples using Ribo-Zero Gold kit (Illumina) according to manufacturer's instructions. Briefly, magnetic beads were washed, samples were treated with rRNA removal solution, rRNA was removed, and rRNA-depleted samples were purified.

\section{RNA Quantification}

RNA was quantified using a Qubit ${ }^{\circledR} 2.0 \quad$ Fluorometer (ThermoFisher Scientific) and Nanodrop Spectrophotometer (ThermoFisher Scientific). The Qubit ${ }^{\circledR}$ assay uses a Qubit RNA BR (Broad-Range) Assay Kit (ThermoFisher Scientific).

\section{Poly(A)-Tailing of RNA Using E. coli Poly(A) Polymerase (EPAP)}

To obtain high quality RNA for further preparation, extracted RNA of each virus received from a different independent lab

\footnotetext{
${ }^{1}$ https://www.atcc.org/products/all/VR-84.aspx

${ }^{2}$ https://www.sigmaaldrich.com/catalog/product/roche/10165948001?lang=en\& region $=\mathrm{US}$
} 
was purified and concentrated using AMPureXP beads (Beckman Coulter) and eluted in $15 \mu$ l nuclease-free water (NEB). Purified and concentrated RNA was incubated at $70^{\circ} \mathrm{C}$ for $2 \mathrm{~min}$ and immediately placed on ice to avoid secondary structure formation. Poly(A)-tailing reaction using E. coli EPAP (NEB\# M0276) was designed to perform in a $26 \mu l$ reaction (reaction size can be scaled up as needed). Purified RNA was treated with $2.5 \mu \mathrm{l}$ 10X E. coli Poly(A) Polymerase Reaction Buffer (supplied with the enzyme), $4.5 \mu \mathrm{l}$ ATP (10 mM) (supplied with the enzyme), and $4 \mu \mathrm{IEPAP}(\mathrm{NEB})$ at $37^{\circ} \mathrm{C}$ for $10 \mathrm{~min}$. Poly(A)-tailed RNA was then cleaned using $2.5 \times$ (by volume) AMPureXP beads (Beckman Coulter) and eluted with 11-15 $\mu$ l nuclease free water (NEB), 2 min at room temperature. Cleaned Poly(A)-tailed RNA was incubated for $1 \mathrm{~min}$ at $70^{\circ} \mathrm{C}$ and immediately placed on ice to remove secondary structure. The cleaned Poly(A)-tailed RNA was used as input for dRNA-seq library preparation.

\section{Library Preparation and Direct RNA Sequencing by Nanopore}

Direct RNA sequencing was performed using the Direct RNA Sequencing Protocol (SQK-RNA001 kit) for the MinION. Some steps of the manufacture protocol were modified. First, the ligation reaction of RNA and adaptor was extended from 10 to $15 \mathrm{~min}$. At this step, control RNA (RCS) was not added. Second, the RT (50 min) step was skipped in our protocol. Third, wirebored tips (Axygen) were used for mixing AMPureXP beads (Beckman Coulter)-RNA. RNA library was eluted and loaded onto a flow cell for sequencing. Sequencing of the RNA was performed on a single R9.4/FLO-MIN106 flow cell on a MinION Mk1B for $\sim 8 \mathrm{~h}$.

\section{Bioinformatics and Real-Time Analysis}

Immediately after loading the library onto a flow cell and sequencing, base-calling was started using the localbased software, Albacore version 2.3.1 (ONT) with the (-disable_filtering-q 0) command modifications from default. We first filtered out the sequenced reads below a size of 200 bases to avoid short, low-quality reads. The remaining reads were mapped to viral genomes and the host genomes using Minimap2 version 2.9 ( $\mathrm{Li}, 2018)$ with the $-\mathrm{k} 7$ from virus and -k 14 for host command modifications from default. The aligned reads were processed in downstream analysis. The number of

\section{REFERENCES}

Carrasco-Hernandez, R., Jacome, R., Lopez Vidal, Y., and Ponce de Leon, S. (2017). Are RNA viruses candidate agents for the next global pandemic? A review. ILAR J. 58, 343-358. doi: 10.1093/ilar/ilx026

Dapprich, J., Ferriola, D., Mackiewicz, K., Clark, P. M., Rappaport, E., D’Arcy, M., et al. (2016). The next generation of target capture technologies - large DNA fragment enrichment and sequencing determines regional genomic variation of high complexity. BMC Genomics 17:486. doi: 10.1186/s12864-016-2836-6

DeFilippis, V., Raggo, C., Moses, A., and Fruh, K. (2003). Functional genomics in virology and antiviral drug discovery. Trends Biotechnol. 21, 452-457. doi: 10.1016/S0167-7799(03)00207-5

Eckert, S. E., Chan, J. Z., Houniet, D., PATHSEEK consortium, Breuer, J., and Speight, G. (2016). Enrichment by hybridisation of long DNA fragments for Nanopore sequencing. Microb. Genom 2:e000087. doi: 10.1099/mgen.0.000087 mapped reads in virus and hosts were measured by Samtools version 1.5 ( $\mathrm{Li}$ et al., 2009). The read coverage was calculated using genomecov package in BEDtools version 2.27.1 (Quinlan and Hall, 2010) and visualized using IGV (Robinson et al., 2011). The reference-based assembly was performed using four rounds consensus polishing of the reference genomes by Racon version 1.2.1 (Vaser et al., 2017).

\section{DATA AVAILABILITY}

The sequence native RNA data of six viruses has been submitted to GenBank and is publicly available under the Bioproject number PRJNA493665.

\section{AUTHOR CONTRIBUTIONS}

IN and TW designed and conceived the project. IN, CJ, and DU supervised the study. CJ, MT, JL, AT, JP, BK, NA, AS, DC, $\mathrm{CC}, \mathrm{MW}, \mathrm{WK}, \mathrm{RS}$, and $\mathrm{NH}$ performed viral cultivation and RNA extraction. TDW and TW optimized library preparation protocol. TW and SK conducted MinION sequencing. PJ and IN performed computational analysis. All authors participated in interpreting the results and writing the manuscript, and read and approved the final version of this manuscript.

\section{FUNDING}

This work was funded in part by the Helen Adams \& Arkansas Research Alliance Endowed Chair, the National Institute of General Medical Sciences of the National Institutes of Health award no. P20GM125503 and National Institutes of Health award no R01AI103053.

\section{SUPPLEMENTARY MATERIAL}

The Supplementary Material for this article can be found online at: https://www.frontiersin.org/articles/10.3389/fmicb. 2019.00260/full\#supplementary-material

Faria, N. R., Quick, J., Claro, I. M., Theze, J., de Jesus, J. G., Giovanetti, M., et al. (2017). Establishment and cryptic transmission of Zika virus in Brazil and the Americas. Nature 546, 406-410. doi: 10.1038/nature 22401

Garalde, D. R., Snell, E. A., Jachimowicz, D., Sipos, B., Lloyd, J. H., Bruce, M., et al. (2018). Highly parallel direct RNA sequencing on an array of nanopores. Nat. Methods 15, 201-206. doi: 10.1038/nmeth.4577

Hoenen, T., Groseth, A., Rosenke, K., Fischer, R. J., Hoenen, A., Judson, S. D., et al. (2016). Nanopore sequencing as a rapidly deployable ebola outbreak tool. Emerg. Infect. Dis. 22, 331-334. doi: 10.3201/eid2202.151796

Jenjaroenpun, P., Wongsurawat, T., Pereira, R., Patumcharoenpol, P., Ussery, D. W., Nielsen, J., et al. (2018). Complete genomic and transcriptional landscape analysis using third-generation sequencing: a case study of Saccharomyces cerevisiae CEN.PK113-7D. Nucleic Acids Res. 4:e38. doi: 10. 1093/nar/gky014 
Karamitros, T., and Magiorkinis, G. (2018). Multiplexed targeted sequencing for oxford nanopore MinION: a detailed library preparation procedure. Methods Mol. Biol. 1712, 43-51. doi: 10.1007/978-1-4939-7514-3_4

Keller, M. W., Rambo-Martin, B. L., Wilson, M. M., Ridenour, C. A., Shepard, S. S., Stark, T. J., et al. (2018). Direct RNA sequencing of the coding complete influenza A virus genome. Sci. Rep. 8:14408. doi: 10.1038/s41598-01832615-8

Kilianski, A., Roth, P. A., Liem, A. T., Hill, J. M., Willis, K. L., Rossmaier, R. D., et al. (2016). Use of unamplified RNA/cDNA-hybrid nanopore sequencing for rapid detection and characterization of RNA viruses. Emerg. Infect. Dis. 22, 1448-1451. doi: 10.3201/eid2208.160270

Li, H. (2018). Minimap2: pairwise alignment for nucleotide sequences. Bioinformatics 34, 3094-3100. doi: 10.1093/bioinformatics/bty191

Li, H., Handsaker, B., Wysoker, A., Fennell, T., Ruan, J., Homer, N., et al. (2009). The Sequence Alignment/Map format and SAMtools. Bioinformatics 25, 20782079. doi: 10.1093/bioinformatics/btp352

Lichinchi, G., Zhao, B. S., Wu, Y., Lu, Z., Qin, Y., He, C., et al. (2016). Dynamics of human and viral RNA methylation during Zika Virus infection. Cell Host. Microbe 20, 666-673. doi: 10.1016/j.chom.2016. 10.002

Marston, D. A., McElhinney, L. M., Ellis, R. J., Horton, D. L., Wise, E. L., Leech, S. L., et al. (2013). Next generation sequencing of viral RNA genomes. BMC Genomics 14:444. doi: 10.1186/1471-2164-14-444

Quinlan, A. R., and Hall, I. M. (2010). BEDTools: a flexible suite of utilities for comparing genomic features. Bioinformatics 26, 841-842. doi: 10.1093/ bioinformatics/btq033
Robinson, J. T., Thorvaldsdottir, H., Winckler, W., Guttman, M., Lander, E. S., Getz, G., et al. (2011). Integrative genomics viewer. Nat. Biotechnol. 29, 24-26. doi: $10.1038 /$ nbt. 1754

Vaser, R., Sovic, I., Nagarajan, N., and Sikic, M. (2017). Fast and accurate de novo genome assembly from long uncorrected reads. Genome Res. 27, 737-746. doi: $10.1101 /$ gr.214270.116

Wongsurawat, T., Jenjaroenpun, P., Wassenaar, T. M., Wadley, T. D., Wanchai, V., Akel, N. N., et al. (2018). Decoding the epitranscriptional landscape from native RNA sequences. bioRXiv [Preprint]. doi: 10.1101/487819

Woolhouse, M. E., Brierley, L., McCaffery, C., and Lycett, S. (2016). Assessing the epidemic potential of RNA and DNA viruses. Emerg. Infect. Dis. 22, 2037-2044. doi: 10.3201/eid2212.160123

Conflict of Interest Statement: The authors declare that the research was conducted in the absence of any commercial or financial relationships that could be construed as a potential conflict of interest.

Copyright (C) 2019 Wongsurawat, Jenjaroenpun, Taylor, Lee, Tolardo, Parvathareddy, Kandel, Wadley, Kaewnapan, Athipanyasilp, Skidmore, Chung, Chaimayo, Whitt, Kantakamalakul, Sutthent, Horthongkham, Ussery, Jonsson and Nookaew. This is an open-access article distributed under the terms of the Creative Commons Attribution License (CC BY). The use, distribution or reproduction in other forums is permitted, provided the original author(s) and the copyright owner(s) are credited and that the original publication in this journal is cited, in accordance with accepted academic practice. No use, distribution or reproduction is permitted which does not comply with these terms. 